\title{
Photochemical synthesis and catalytic applications of gold nanoplates fabricated using Quercetin Diphosphate macromolecules
}

Francis J. Osonga, ${ }^{1}$ Victor M. Kariuki, ${ }^{1}$ Victor M. Wambua, ${ }^{1}$ Sanjay Kalra, ${ }^{1}$ Bruno Nweke, ${ }^{1}$ Roland M.Miller, ${ }^{1}$ Mustafa Çeşme ${ }^{1} \&$ *Omowunmi A. Sadik ${ }^{1}$

\author{
${ }^{1}$ Department of Chemistry \\ Center for Research in Advanced Sensing Technologies \& Environmental Sustainability \\ (CREATES) \\ State University of New York at Binghamton \\ P.O Box 6000 Binghamton, NY, 13902.
}

Corresponding Author: osadik@binghamton.edu

Osonga et al

Page S1 
Supporting Information Content:

- Computed images of nine of the other conformers that could be taking place

- Determination of the $\Delta \mathrm{G}$ and $\Delta \mathrm{H}$ for each conformer

- The computed energy for each conformer

- TEM images of P, Q, R, S, T, U with calculated edge length

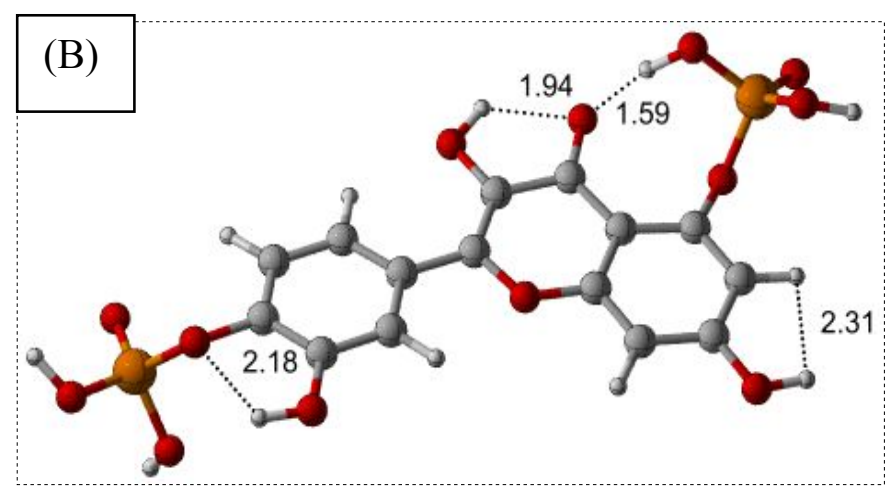

\section{Conformer $2 \Delta G=1.3 \mathrm{kcal} / \mathrm{mol}$ $\Delta \mathrm{H}=2 \mathrm{kcal} / \mathrm{mol}$}

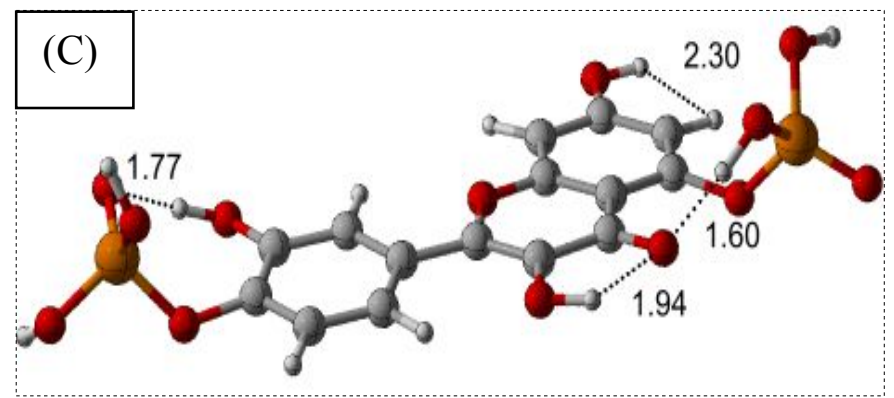

\section{Conformer $3 \Delta \mathrm{G}=0.17 \mathrm{kcal} / \mathrm{mol}$ $\Delta \mathrm{H}=0.012 \mathrm{Kcal} / \mathrm{mol}$}

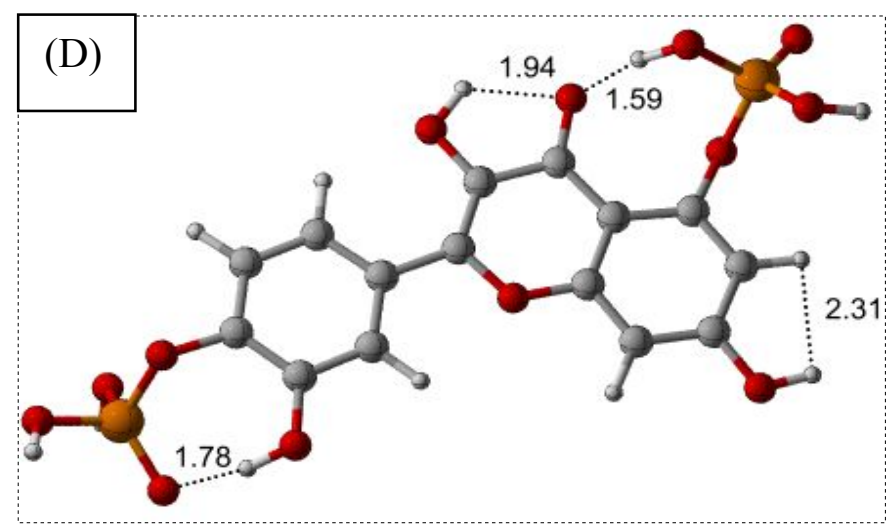

Conformer $4 \Delta \mathrm{G}=0.223 \mathrm{kcal} / \mathrm{mol}$
$\Delta \mathrm{H}=0.03 \mathrm{Kcal}$




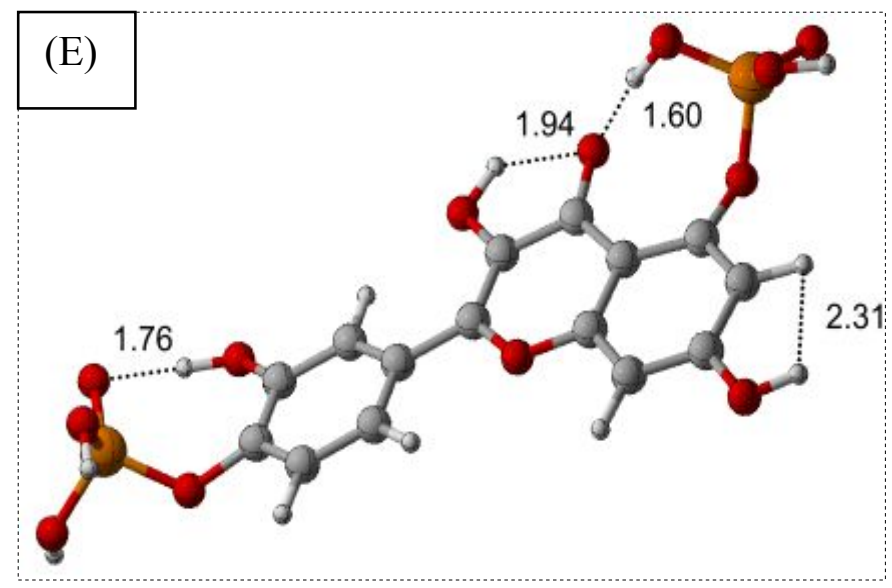

\section{Conformer $5 \Delta G=0.47 \mathrm{kcal} / \mathrm{mol}$ $\Delta \mathrm{H}=0.43 \mathrm{kcal} / \mathrm{mol}$}
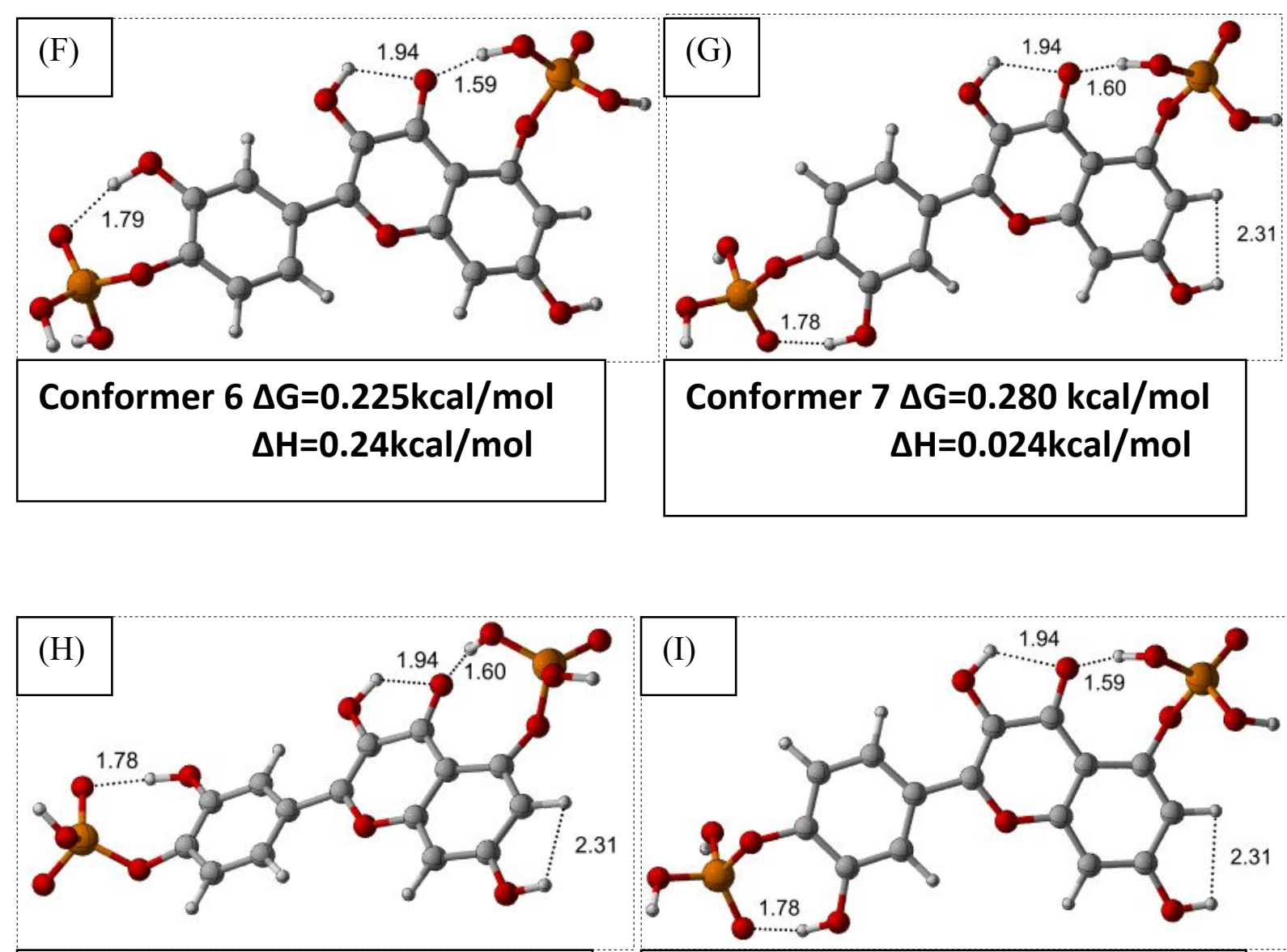

Conformer $8 \Delta G=0.65 \mathrm{kcal} / \mathrm{mol}$ $\Delta \mathrm{H}=0.076 \mathrm{kcal} / \mathrm{mol}$ 


\section{COMPUTED STRUCTURES AND ENERGIES}

\section{Conformer 1 (A)}

\section{Energy $=-2239.411349$}

\begin{tabular}{|c|c|c|c|}
\hline $\mathrm{C}$ & 4.52049200 & 1.94587300 & 0.40627600 \\
\hline $\mathrm{C}$ & 4.08030000 & 0.64072600 & 0.47010200 \\
\hline $\mathrm{C}$ & 2.70984800 & 0.30512200 & 0.27683900 \\
\hline $\mathrm{C}$ & 1.82560300 & 1.39127600 & 0.06548400 \\
\hline $\mathrm{C}$ & 2.24981400 & 2.71552800 & -0.0073680 \\
\hline C & 3.60396300 & 2.98864400 & 0.15243900 \\
\hline $\mathrm{H}$ & 5.57015900 & 2.16009200 & 0.57696500 \\
\hline C & 2.14801400 & -1.02400100 & 0.2799530 \\
\hline $\mathrm{H}$ & 1.53519300 & 3.51171900 & -0.1771220 \\
\hline C & -0.09569900 & -0.03388200 & -0.00835600 \\
\hline $\mathrm{C}$ & 0.70343600 & -1.13181700 & 0.178585 \\
\hline C & -1.55048100 & 0.02778400 & -0.1834100 \\
\hline $\mathrm{C}$ & -2.16313700 & 1.26056000 & -0.48899700 \\
\hline C & -2.35620200 & -1.11532900 & -0.04427800 \\
\hline C & -3.53863000 & 1.32564500 & -0.6672550 \\
\hline $\mathrm{H}$ & -1.56763400 & 2.15778100 & -0.5955220 \\
\hline C & -3.74227500 & -1.05828200 & -0.2174550 \\
\hline $\mathrm{H}$ & -1.92542300 & -2.07524200 & 0.2032330 \\
\hline C & -4.32224900 & 0.18220400 & -0.5423510 \\
\hline $\mathrm{H}$ & -4.02283400 & 2.26215800 & -0.9237580 \\
\hline 0 & 0.22424300 & -2.39635100 & 0.225735 \\
\hline $\mathrm{H}$ & 1.03262300 & -2.95403600 & 0.3051870 \\
\hline 0 & 2.78182300 & -2.11681400 & 0.3537720 \\
\hline 0 & 0.49182000 & 1.19802900 & -0.0737590 \\
\hline O & 4.98713900 & -0.32632100 & 0.8599070 \\
\hline 0 & 3.99278600 & 4.28000800 & 0.0766210 \\
\hline $\mathrm{H}$ & 4.95252000 & 4.35190400 & 0.2136190 \\
\hline 0 & -4.43980600 & -2.21649600 & -0.0988660 \\
\hline $\mathrm{H}$ & -5.36894800 & -2.03139600 & 0.1767400 \\
\hline 0 & -5.68292900 & 0.29320600 & -0.8693320 \\
\hline P & 5.97461600 & -1.18433700 & -0.1258000 \\
\hline $\mathrm{P}$ & -6.87341400 & 0.04765500 & 0.1867040 \\
\hline 0 & 6.53458500 & -0.15145900 & -1.2245030 \\
\hline $\mathrm{H}$ & 7.41993900 & 0.17592000 & -0.987329 \\
\hline 0 & 4.98646600 & -2.05154900 & -1.0063060 \\
\hline $\mathrm{H}$ & 4.12382400 & -2.20669100 & -0.4992110 \\
\hline 0 & 7.00107100 & -1.86184800 & 0.6973060 \\
\hline 0 & -6.84842900 & -1.29757900 & 0.8291570 \\
\hline 0 & -8.16484000 & 0.38733600 & -0.6884280 \\
\hline $\mathrm{H}$ & -8.52120000 & -0.39164500 & -1.1525080 \\
\hline 0 & -6.74180300 & 1.31017200 & 1.156229 \\
\hline 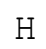 & -7.16003800 & 1.16667700 & 2.024644 \\
\hline
\end{tabular}

Osonga et al

Page S4 
Conformer 2(B) Energy $=-2239.40921$

\begin{tabular}{|c|c|c|c|}
\hline $\mathrm{C}$ & 4.43534000 & 1.91148500 & 0.51997500 \\
\hline C & 4.03685300 & 0.59143300 & 0.51799800 \\
\hline C & 2.67856200 & 0.22254000 & 0.30211700 \\
\hline C & 1.76135100 & 1.28928200 & 0.13793500 \\
\hline $\mathrm{C}$ & 2.14345500 & 2.62820300 & 0.13001100 \\
\hline C & 3.48763100 & 2.93582100 & 0.31012300 \\
\hline $\mathrm{H}$ & 5.47650000 & 2.15035100 & 0.70839800 \\
\hline C & 2.15927100 & -1.12243300 & 0.23828500 \\
\hline $\mathrm{H}$ & 1.40467900 & 3.40863200 & -0.00621200 \\
\hline $\mathrm{C}$ & -0.11338400 & -0.19090300 & -0.01223600 \\
\hline C & 0.71931200 & -1.27102400 & 0.12624500 \\
\hline C & -1.56894200 & -0.16967700 & -0.18929200 \\
\hline C & -2.35010100 & -1.33491500 & -0.05327000 \\
\hline C & -2.20214100 & 1.04904800 & -0.50072400 \\
\hline C & -3.73101600 & -1.27721700 & -0.22779900 \\
\hline $\mathrm{H}$ & -1.88559600 & -2.28054400 & 0.18559000 \\
\hline C & -3.58129600 & 1.10806100 & -0.67184400 \\
\hline $\mathrm{H}$ & -1.63155000 & 1.96205600 & -0.61451200 \\
\hline C & -4.33944300 & -0.06407200 & -0.5262960 \\
\hline $\mathrm{H}$ & -4.34015500 & -2.16809700 & -0.12717400 \\
\hline 0 & 0.27983600 & -2.55082200 & 0.10868800 \\
\hline $\mathrm{H}$ & 1.10520600 & -3.08609100 & 0.16306200 \\
\hline 0 & 2.82764300 & -2.19687900 & 0.26083100 \\
\hline 0 & 0.43474900 & 1.06061000 & -0.01758800 \\
\hline 0 & 4.97354800 & -0.36455200 & 0.8623610 \\
\hline 0 & 3.83603900 & 4.24084700 & 0.2971180 \\
\hline $\mathrm{H}$ & 4.79320900 & 4.33568100 & 0.4381510 \\
\hline 0 & -4.16347300 & 2.29710100 & -0.9779370 \\
\hline $\mathrm{H}$ & -5.11972800 & 2.14757800 & -1.08642000 \\
\hline 0 & -5.70903900 & 0.06968400 & -0.77526300 \\
\hline $\mathrm{P}$ & 5.99232500 & -1.13449100 & -0.16387700 \\
\hline $\mathrm{P}$ & -6.83914700 & -0.41680800 & 0.2895930 \\
\hline 0 & 6.50105200 & -0.03074800 & -1.2178190 \\
\hline $\mathrm{H}$ & 7.36544900 & 0.33582600 & -0.9613020 \\
\hline 0 & 5.03745000 & -2.00399100 & -1.0779350 \\
\hline $\mathrm{H}$ & 4.17705800 & -2.20453900 & -0.5824350 \\
\hline 0 & 7.05162700 & -1.80313600 & 0.6240940 \\
\hline O & -6.80052100 & -1.84166600 & 0.6874050 \\
\hline 0 & -8.16397900 & 0.08241700 & -0.46037200 \\
\hline $\mathrm{H}$ & -8.58172000 & -0.62779100 & -0.97926500 \\
\hline 0 & -6.63926300 & 0.67621100 & 1.4449370 \\
\hline $\mathrm{H}$ & -6.99907500 & 0.38200900 & 2.300843 \\
\hline
\end{tabular}

Conformer $3(\mathrm{C})$

Energy $=-2239.411082$

Osonga et al

Page S5 
C
C
C
C
C
C
H
C
H
C
C
C
C
C
C
H
C
H
C
H
O
H
O
O
O
O
H
O
H
O
P
P
O
H
O
H
O
O
O
H
O
H

Conformer 4 (D) Energy $=-2239.410993$

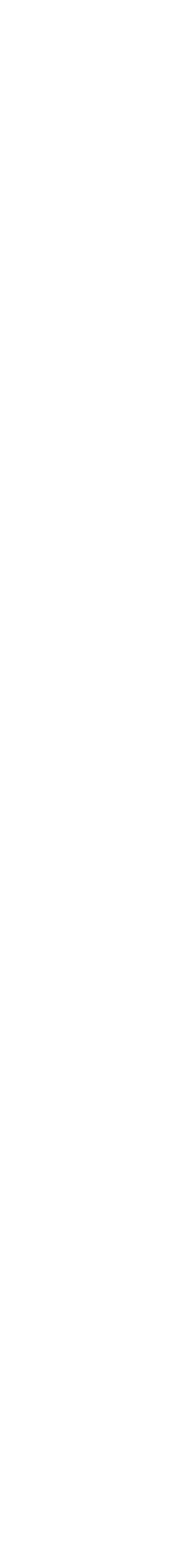

$\begin{array}{lll}4.38883400 & 1.99818700 & -0.26903900\end{array}$

$\begin{array}{lll}4.05432400 & 0.66480700 & -0.37507300\end{array}$

$\begin{array}{lll}2.69859600 & 0.23096400 & -0.33570400\end{array}$

$\begin{array}{lll}1.71971300 & 1.24975800 & -0.23800500\end{array}$

$2.03596000 \quad 2.60092200 \quad-0.12243400$

$\begin{array}{lll}3.37644100 & 2.97098800 & -0.12498100\end{array}$

$\begin{array}{lll}5.43235000 & 2.28926700 & -0.32266600\end{array}$

$2.23893100 \quad-1.13556700 \quad-0.38666800$

$\begin{array}{lll}1.25108200 & 3.34308400 & -0.04135900\end{array}$

$-0.08861900-0.31115100-0.39013800$

0.80453600

$-1.55430600$

$-2.25817000$

$-2.28184100$

$-3.64779300$

$-1.72908400$

$-3.67761700$

$-1.77414100$

$-4.35297200$

$-4.20547500$

0.42886100

1.27975400

2. 95511500

0.39630600

5.06546800

3.66053600

4.62231100

$-4.29578400$

$-5.19849900$

$-5.74391500$

5.99697900

$-6.80601200$

4.97550600

4.18929000

6.38884300

7.26985200

7.13419800

$-6.63320800$

$-6.65652300$

$-7.01063300$

$-8.19682600$

$-8.55604200$
$-1.34875900$

$-0.35667800$

$-1.56269700$

0.83424100

$-1.55001500$

$-2.49520100$

0.85598900

1.77674800

$-0.36134200$

$-2.46527400$

$-2.64521100$

$-3.14125900$

$-2.17876800$

0.95949300

$-0.23390000$

4.28646800

4.42648200

2.05614300

1. 93564800

$-0.40563200$

$-1.01796400$

$-0.05268100$

$-1.92306600$

$-2.15397600$

0.06236900

0.44311200

$-1.64928600$

1.30522400

$-1.29626200$

$-1.11907000$

$-0.32804400$

0.46602300
$-0.46323700$

$-0.41965300$

$-0.61400500$

$-0.24895300$

$-0.64973900$

$-0.74490000$

$-0.27891400$

$-0.08753700$

$-0.49287500$

$-0.81902300$

$-0.55876600$

$-0.53150600$

$-0.36624900$

$-0.25811100$

$-0.65739100$

$-0.00901400$

$-0.02810000$

$-0.14071600$

0.23911200

$-0.67608600$

0.43815500

0.48115800

1.23946400

0.64499500

1.56380200

1.40165700

$-0.26761600$

1.07204900

1.47227800

2. 36279600

$-0.25337300$

$-0.68882700$

$$
\begin{aligned}
& \text { C } \\
& \text { C }
\end{aligned}
$$

C
4.33714700

4.01489800

2. 67769700
2.01311200

0.67333200

0.22097000
0.43738600

0.48490800

0.29791300

Osonga et al

Page S6 
C
C
C
H
C
H
C
C
C
C
C
C
H
C
H
C
H
O
H
O
O
O
O
H
O
H
O
P
P
O
H
O
H
O
O
H
O
O
H

Conformer 5 (E) Energy $=-2239.410606$

C
C
C
C
C
C

$$
\begin{array}{r}
1.69847200 \\
2.00329700 \\
3.33001900 \\
5.36483400 \\
2.23664000 \\
1.21975800 \\
-0.08971500 \\
0.80618900 \\
-1.54644900 \\
-2.24490000 \\
-2.27160900 \\
-3.62480500 \\
-1.71838900 \\
-3.65823100 \\
-1.76897400 \\
-4.32582600 \\
-4.17620700 \\
0.44353400 \\
1.30041500 \\
2.96658400 \\
0.38571900 \\
5.00763300 \\
3.60381400 \\
4.55684900 \\
-4.27365400 \\
-5.20636000 \\
-5.69366000 \\
6.05712900 \\
-6.85186900 \\
6.51059400 \\
7.35574100 \\
5.14064700 \\
4.30106400 \\
7.14795600 \\
-8.17342500 \\
-8.46413100 \\
-6.71329000 \\
-6.81473000 \\
-7.20222600
\end{array}
$$

1.22744000

2.58488400

2.97390100

2.31763700

$-1.15257300$

3. 31704300

$-0.36203000$

$-1.38758500$

$-0.43015100$

$-1.65350500$

0.75684000

$-1.66660400$

$-2.58169300$

0.75399700

1.71493800

$-0.48547900$

$-2.60088200$

$-2.69088000$

$-3.17356300$

$-2.18536500$

0.91798500

$-0.21609700$

4.29504600

4.44787600

1.94206900

1.91020400

$-0.58888400$

$-0.96153800$

0.02523500

0.14019300

0.55536000

$-1.89317300$

$-2.13643900$

$-1.56299100$

$-0.43470600$

0.21145400

1. 48724100

$-0.93654300$

$-0.52785600$
0.11300200

0.05468500

0.20406100

0.60410300

0.28067900

$-0.09833800$

0.03273300

0.19236400

$-0.12335800$

$-0.06596500$

$-0.32909800$

$-0.23425000$

0.10053100

$-0.49223100$

$-0.36731500$

$-0.45351200$

$-0.21307300$

0.22491100

0.28614000

0.32708400

$-0.01704600$

0.85014200

0.13872800

0.25439500

$-0.71989400$

$-0.39953200$

$-0.75074300$

$-0.16317200$

0.18472300

$-1.24403700$

$-0.99759500$

$-1.05538300$

$-0.54460900$

0.63574900

$-0.58412700$

$-1.25281100$

0.44221600

1.45923000

2. 25430900

Osonga et al

Page S7

$-4.59758600$

$-4.12772000$

$-2.73464500$

$-1.86572500$

$-2.31925000$

$-3.69055100$
1.88762600

0.59452800

0.30219600

1. 41722900

2.72994500

2. 96093300
$-0.29292200$

$-0.38433500$

$-0.34465800$

$-0.25670800$

$-0.15644600$

$-0.16221200$ 
$\mathrm{H}$
$\mathrm{C}$
$\mathrm{H}$
$\mathrm{C}$
$\mathrm{C}$
$\mathrm{C}$
$\mathrm{C}$
$\mathrm{C}$
$\mathrm{C}$
$\mathrm{H}$
$\mathrm{C}$
$\mathrm{H}$
$\mathrm{C}$
$\mathrm{H}$
$\mathrm{O}$
$\mathrm{H}$
$\mathrm{O}$
$\mathrm{O}$
$\mathrm{O}$
$\mathrm{O}$
$\mathrm{H}$
$\mathrm{O}$
$\mathrm{H}$
$\mathrm{O}$
$\mathrm{P}$
$\mathrm{P}$
$\mathrm{O}$
$\mathrm{H}$
$\mathrm{O}$
$\mathrm{H}$
$\mathrm{O}$
$\mathrm{O}$
$\mathrm{H}$
$\mathrm{O}$
$\mathrm{O}$
$\mathrm{H}$

$\mathrm{H}$

C

C

C

C

C

H

H

O

$\mathrm{P}$

$\mathrm{P}$

O

$\mathrm{H}$

O

$\mathrm{H}$

O

O

$\mathrm{H}$

O

$\mathrm{O}$

\section{Conformer $6(\mathrm{~F})$}

\section{Energy $=-2239.41099$}

$\mathrm{C}$
$\mathrm{C}$
$\mathrm{C}$
$\mathrm{C}$
$\mathrm{C}$
$\mathrm{C}$
$\mathrm{H}$
$\mathrm{C}$
$\mathrm{H}$
$-5.66590600$

$-2.13688600$

$-1.61468800$

0.09269100

$-0.68821600$

1.55542000

2.16356100

2.37148800

3.54786200

1.55890200

3.76631000

1.94170100

4.34454400

4.03114700

$-0.17804400$

$-0.97135300$

$-2.74170500$

$-0.51940300$

$-5.04323800$

$-4.10746800$

$-5.07860700$

4.47624800

5.36615200

5.72908100

$-5.88053100$

6.81339500

$-6.35856600$

$-7.27493000$

$-4.76602200$

$-3.96610600$

$-6.96150700$

8.17221100

8.48245700

6.72838900

6.62584700

6.66391100
2.06818200

$-1.01062900$

3.54950900

0.04884100

$-1.07511000$

0.15377700

1.39204500

$-0.95694000$

1.49906700

2.26266000

$-0.85956800$

$-1.92281200$

0.39189300

2.44835900

$-2.32589200$

$-2.90903500$

$-2.12171200$

1.26482600

$-0.40664700$

4.24179100

4.28258300

$-1.97265300$

$-1.94200900$

0.60517000

$-1.26496000$

$-0.09362000$

$-0.21482300$

0.07636400

$-2.05960800$

$-2.21497500$

$-2.01067100$

0.57426700

0.23361300

$-1.57895500$

0.51753300

1.49026600
$-0.34634200$

$-0.38803800$

$-0.08310800$

$-0.38798400$

$-0.46306100$

$-0.38324700$

$-0.09250400$

$-0.65642400$

$-0.08674600$

0.12552300

$-0.65084200$

$-0.88183300$

$-0.36580300$

0.12044700

$-0.54475800$

$-0.50948400$

$-0.35754300$

$-0.27116700$

$-0.64835000$

$-0.06073200$

$-0.07493900$

$-0.96369200$

$-0.53701200$

$-0.46298300$

0.46801700

0.51338200

1.58898200

1.43761200

1.26217800

0.66149400

$-0.21414100$

0.00828900

$-0.85016100$

0.51958300

1. 97334100

2.01634100

Osonga et al

Page S8

4.51536200

4.07674700

2.70666100

1. 82123000

2.24387500

3.59760400

5.56470700

2.14633200

1.52833200
1.94599600

0.64002600

0.30368000

1.38973100

2.71479500

2.98883700

2.16075300

$-1.02613700$

3. 51087800
0.41565400

0.47336800

0.27872600

0.07172600

0.00498700

0.16666600

0.58757500

0.27677000

$-0.16134900$ 


\section{Conformer $7(\mathrm{G})$}

\section{Energy $=-2239.410903$}

$$
\begin{array}{r}
-0.09822100 \\
0.70179800 \\
-1.55276900 \\
-2.16402600 \\
-2.35884000 \\
-3.53923600 \\
-1.56762800 \\
-3.74473400 \\
-1.92847900 \\
-4.32290700 \\
-4.02268900 \\
0.22354300 \\
1.03226500 \\
2.78127800 \\
0.48767700 \\
4.98482200 \\
3.98480400 \\
4.94435200 \\
-4.44183700 \\
-5.37856800 \\
-5.68432500 \\
5.97351000 \\
-6.87051200 \\
6.52745500 \\
7.41222400 \\
4.98657600 \\
4.12394800 \\
7.00419400 \\
-6.68309500 \\
-7.08250800 \\
-6.88638000 \\
-8.17069700 \\
-8.25631900
\end{array}
$$

$$
\begin{array}{r}
-0.00836400 \\
0.17505300 \\
-0.18601300 \\
-0.51017000 \\
-0.03166500 \\
-0.69125200 \\
-0.62890000 \\
-0.20740300 \\
0.22982700 \\
-0.55055700 \\
-0.96175200 \\
0.21650200 \\
0.29323600 \\
0.34595600 \\
-0.06877800 \\
0.85827000 \\
0.09737100 \\
0.23535200 \\
-0.07200200 \\
0.17611300 \\
-0.87829200 \\
-0.13194700 \\
0.18394500 \\
-1.22861900 \\
-0.99244700 \\
-1.01265400 \\
-0.50590300 \\
0.68687800 \\
1.18105400 \\
2.05688800 \\
0.80821900 \\
-0.70108300 \\
-1.03633700
\end{array}
$$

\author{
C
}

C

C

C

C

C

$\mathrm{H}$

C

$\mathrm{H}$
4.33716700

4.01484400

2.67765600

1.69851600

2.00341700

3.33012200

5.36485200

2.23649500

1.21993100
2.01316100

0.67340000

0.22104000

1.22752900

2. 58495500

2. 97394400

2. 31762700

$-1.15251800$

3. 31711300
0.43722900

0.48495800

0.29777900

0.11254400

0.05406200

0.20360500

0.60407600

0.28068700

$-0.09923900$

Osonga et al

Page S9 


$\begin{array}{rrr}-0.08977900 & -0.36182700 & 0.03210200 \\ 0.80599700 & -1.38740700 & 0.19217000 \\ -1.54650200 & -0.42981300 & -0.12414200 \\ -2.24494300 & -1.65323100 & -0.06807100 \\ -2.27168300 & 0.75738600 & -0.32871700 \\ -3.62484900 & -1.66621800 & -0.23647800 \\ -1.71841500 & -2.58158300 & 0.09747000 \\ -3.65826200 & 0.75463200 & -0.49200500 \\ -1.76907600 & 1.71554000 & -0.36588400 \\ -4.32585500 & -0.48488000 & -0.45458000 \\ -4.17620900 & -2.60054400 & -0.21631100 \\ 0.44323100 & -2.69065900 & 0.22515400 \\ 1.30007400 & -3.17337900 & 0.28674700 \\ 2.96632100 & -2.18533400 & 0.32746500 \\ 0.38577400 & 0.91815700 & -0.01777700 \\ 5.00756700 & -0.21588200 & 0.85057000 \\ 3.60398500 & 4.29508000 & 0.13811200 \\ 4.55701700 & 4.44786300 & 0.25387100 \\ -4.27389300 & 1.94281000 & -0.71876900 \\ -5.20608800 & 1.91079400 & -0.39692800 \\ -5.69374200 & -0.58781200 & -0.75182700 \\ 6.05664300 & -0.96199800 & -0.16266100 \\ -6.85136800 & 0.02482500 & 0.18543500 \\ 6.51303900 & 0.13989200 & -1.24215900 \\ 7.35819200 & 0.55401300 & -0.99396600 \\ 5.13928500 & -1.89079900 & -1.05701900 \\ 4.30059400 & -2.13577700 & -0.54589700 \\ 7.14554300 & -1.56624800 & 0.63673600 \\ -8.17334700 & -0.43289000 & -0.58400000 \\ -8.46167300 & 0.21273200 & -1.25423500 \\ -6.71170900 & 1.48610300 & 0.44642600 \\ -6.81514300 & -0.93962700 & 1.45794700 \\ -7.19767600 & -0.53007100 & 2.25497300\end{array}$

\section{Conformer $8(\mathrm{H})$}

\section{Energy $=-2239.410319$}

$\mathrm{C}$
$\mathrm{C}$
$\mathrm{C}$
$\mathrm{C}$
$\mathrm{C}$
$\mathrm{C}$
$\mathrm{H}$
$\mathrm{C}$
$\mathrm{H}$
$\mathrm{C}$
$\mathrm{C}$
$\mathrm{C}$

-4.59562500
-4.12510500
-2.73193800
-1.86355800
-2.31774800
-3.68913900
-5.66395700
-2.13360800
-1.61361600
0.09556100
-0.68487200
1.55825900

1.88639500

$-0.29076000$

$-0.38392000$

0.59370500

0.30191700

$-0.34342300$

1. 41717900

$-0.25314100$

2. 72953000

$-0.15098000$

2.95990100

$-0.15737800$

2.06667700

$-0.34491100$

$-1.01054900$

$-0.38779500$

3. 54929600

$-0.07577400$

0.04987500

$-0.38547500$

$-1.07434700$

$-0.46183100$

$-0.38191300$

Osonga et al

Page S10 
2.16567500

2. 37473300

3. 54985500

1.56033100

3. 76931500

1. 94550800

4.34628700

4.03315700

$-0.17426000$

$-0.96750900$

$-2.73792200$

$-0.51719800$

$-5.03967400$

$-4.10675100$

$-5.07789200$

4. 48017200

5.37517200

5.73048800

$-5.87875600$

6.80512900

$-6.35628400$

$-7.27218600$

$-4.76550500$

$-3.96419000$

$-6.96019700$

8.17051200

8.62026000

6.76689000

6.50808700

6.83388700
1. 39552200

$-0.95522000$

1. 50445200

2. 26585900

$-0.85566900$

$-1.92224400$

0.39753200

2. 45402300

$-2.32489200$

$-2.90823800$

$-2.12200100$

1. 26536000

$-0.40764200$

4. 24035400

4.28068100

$-1.97007800$

$-1.93351900$

0.60834100

$-1.26746300$

$-0.07897000$

$-0.21933800$

0.07336800

$-2.06527900$

$-2.21775100$

$-2.01045400$

0.59230000

0.07410100

$-1.56943500$

0.64503100

0.14000300
$-0.09540000$

$-0.65282100$

$-0.09263600$

0.12180300

$-0.64981600$

$-0.87426600$

$-0.37151100$

0.11241300

$-0.54478900$

$-0.51120700$

$-0.35929700$

$-0.26716100$

$-0.65061600$

$-0.05403400$

$-0.06870200$

$-0.95797300$

$-0.54460700$

$-0.47574000$

0.46324600

0.50703100

1. 58615200

1.43507200

1.25602400

0.65625600

$-0.22117400$

0.02341800

$-0.66794600$

0.50241600

1.89953000

2.66644400

\section{Conformer 9 (I)}

\section{Energy $=-2239.410983$}

$$
\begin{array}{r}
4.33717200 \\
4.01490000 \\
2.67769300 \\
1.69849200 \\
2.00334000 \\
3.33006400 \\
5.36486700 \\
2.23660300 \\
1.21981900 \\
-0.08972700 \\
0.80615100 \\
-1.54646100 \\
-2.24489400 \\
-2.27162800
\end{array}
$$$$
2.01312500
$$$$
0.67335200
$$$$
0.22100900
$$$$
1.22749400
$$$$
2.58493100
$$$$
2.97393100
$$$$
\text { 2. } 31763000
$$$$
-1.15252200
$$$$
\text { 3.31710400 }
$$$$
-0.36194500
$$$$
-1.38750900
$$$$
-0.43004600
$$$$
-1.65342100
$$

0.43736400

0.48491200

0.29790600

0.11294300

0.05461200

0.20401700

0.60407200

0.28074100

$-0.09844000$

0.03270900

0.19242200

$-0.12342000$

$-0.06628300$

0.75697800

Osonga et al

Page S11 


$\begin{array}{lrrr}\mathrm{C} & -3.62479700 & -1.66652000 & -0.23461800 \\ \mathrm{H} & -1.71836900 & -2.58163000 & 0.10005000 \\ \mathrm{C} & -3.65824000 & 0.75413200 & -0.49214800 \\ \mathrm{H} & -1.76900600 & 1.71509000 & -0.36697900 \\ \mathrm{C} & -4.32581200 & -0.48536100 & -0.45368700 \\ \mathrm{H} & -4.17617900 & -2.60081400 & -0.21364100 \\ \mathrm{O} & 0.44346700 & -2.69079700 & 0.22503700 \\ \mathrm{H} & 1.30032100 & -3.17352600 & 0.28628700 \\ \mathrm{O} & 2.96651200 & -2.18533800 & 0.32721100 \\ \mathrm{O} & 0.38574000 & 0.91805600 & -0.01715500 \\ \mathrm{O} & 5.00763200 & -0.21607500 & 0.85014000 \\ \mathrm{O} & 3.60388600 & 4.29506400 & 0.13862500 \\ \mathrm{H} & 4.55690500 & 4.44789100 & 0.25442500 \\ \mathrm{O} & -4.27372000 & 1.94221400 & -0.71964000 \\ \mathrm{H} & -5.20634800 & 1.91029700 & -0.39904400 \\ \mathrm{O} & -5.69365100 & -0.58867100 & -0.75099800 \\ \mathrm{P} & 6.05704200 & -0.96164300 & -0.16314900 \\ \mathrm{P} & -6.85176900 & 0.02512700 & 0.18479000 \\ \mathrm{O} & 6.51057200 & 0.13999000 & -1.24407600 \\ \mathrm{H} & 7.35572100 & 0.55515800 & -0.99765300 \\ \mathrm{O} & 5.14048800 & -1.89324900 & -1.05531000 \\ \mathrm{H} & 4.30090900 & -2.13647500 & -0.54448500 \\ \mathrm{O} & 7.14783800 & -1.56313400 & 0.63579400 \\ \mathrm{O} & -8.17341000 & -0.43445000 & -0.58413700 \\ \mathrm{H} & -8.46412400 & 0.21198400 & -1.25255400 \\ \mathrm{O} & -6.71310000 & 1.48702700 & 0.44284800 \\ \mathrm{O} & -6.81458600 & -0.93713600 & 1.45893100 \\ \mathrm{H} & -7.20209300 & -0.52874000 & 2.25415500\end{array}$

Figure S1: Computed optimized QDP structures (B-I) and energies for conformers 1 to 9(A-I). 

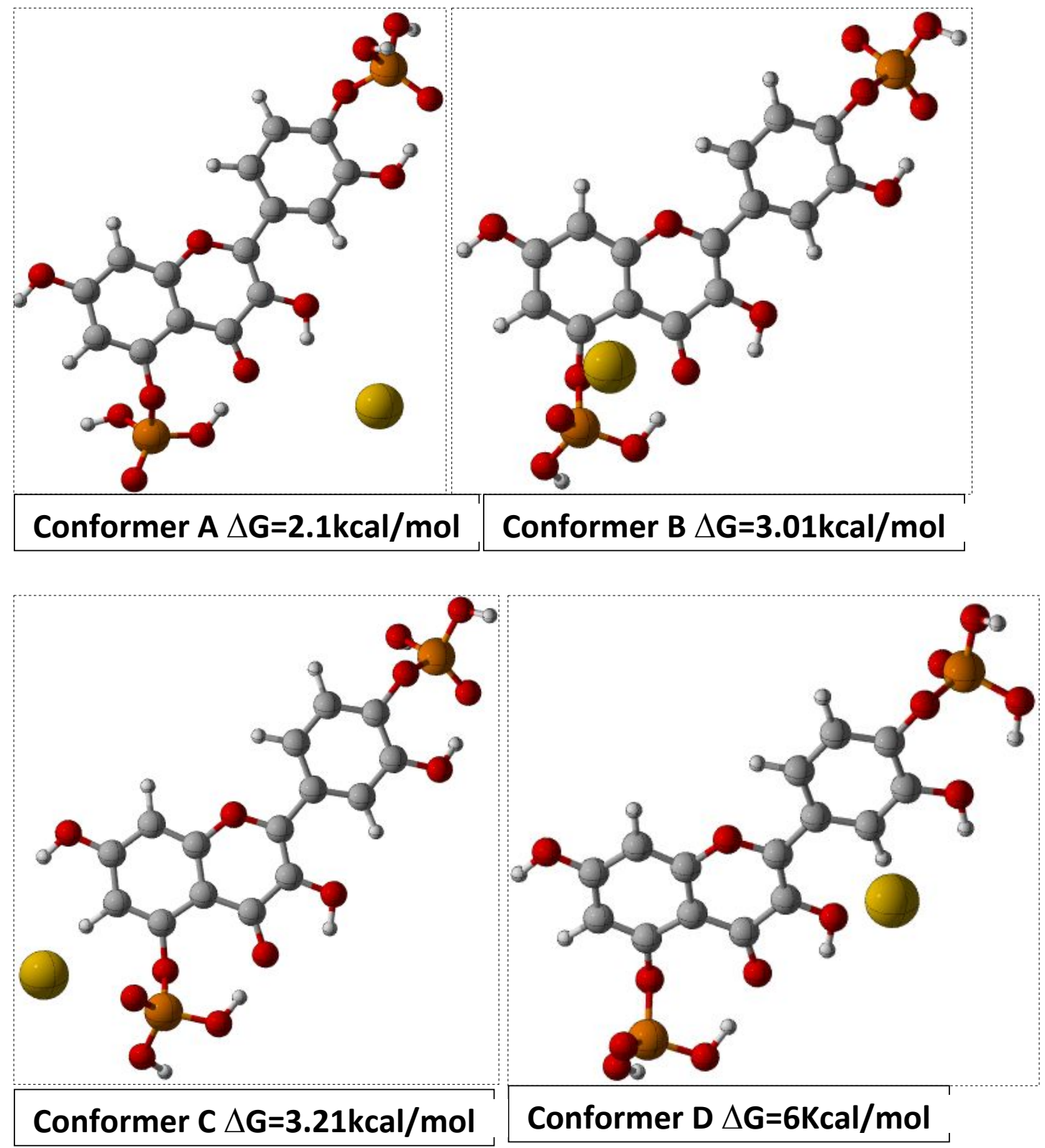

Osonga et al

Page S13 

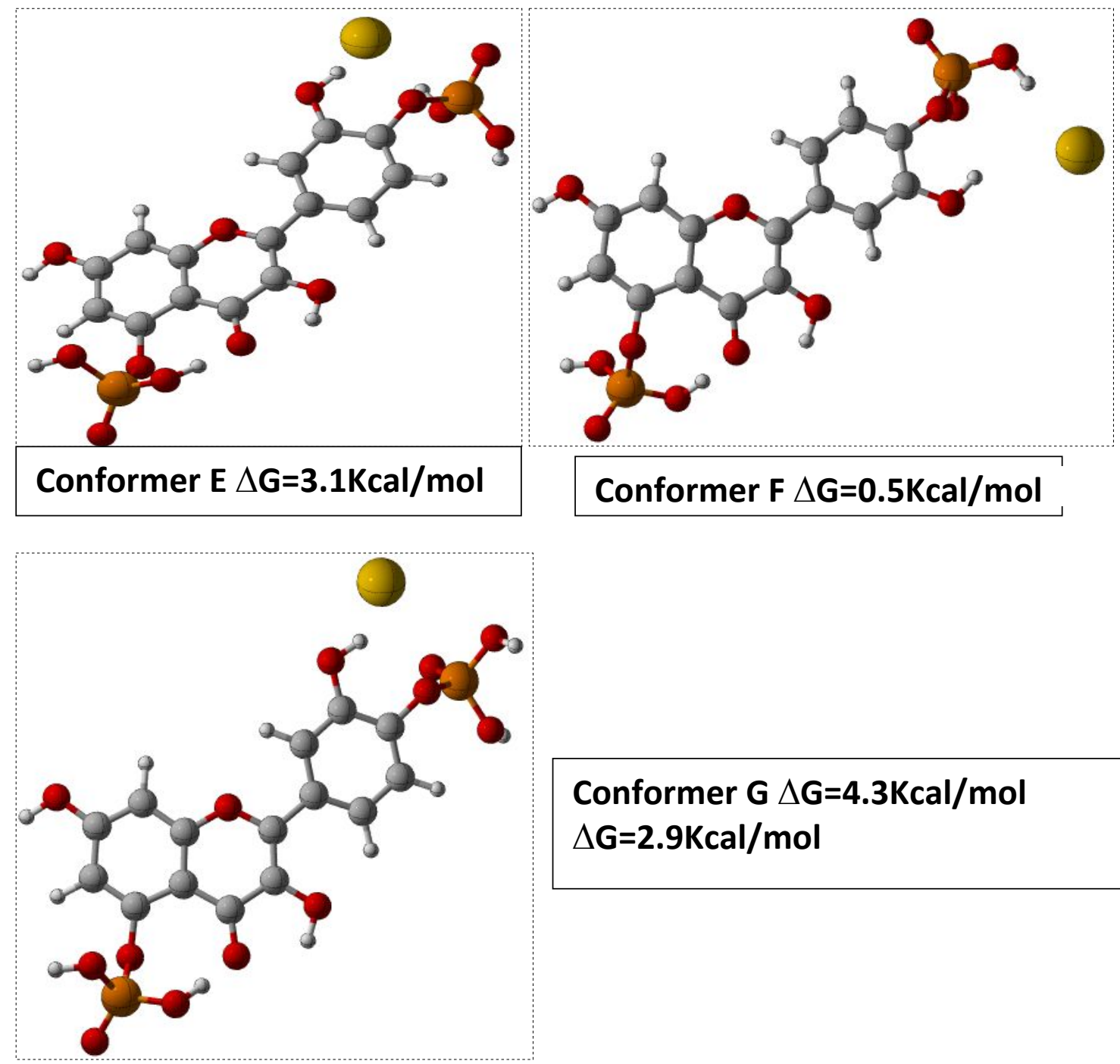

\section{Conformer $\mathbf{G} \Delta \mathbf{G}=4.3 \mathrm{Kcal} / \mathrm{mol}$ $\Delta \mathbf{G}=2.9 \mathrm{Kcal} / \mathrm{mol}$}

\section{COMPUTED STRUCTURES AND ENERGIES}

Conformer 1(A)
C
7.23505900
1.62493800
$-0.16708000$
6.65066300
0.42228500
0.24535500
0.17467800
5.23632700
1.38402600
0.15866400
4.47372900
2.60312100
$-0.20517600$
5.04289200
2.71671700
$-0.56849300$
6.42800200
1.72330000
$-0.55286900$
8.31452100
4.51563500
$-0.95847700$
$-0.11662600$
4.41374300
3. 44145700
0.50160800
2.39693900
0.25852700
$-0.84194200$
3. 06877900
$-0.88214600$
0.15936800
0.94921500
0.48462300
0.51920700
0.45590300
1. 60812200
0.08317300
$-0.61360000$

Osonga et al

Page S14 
C
C
H
C
H
C
H
O
H
O
O
$\mathrm{O}$
$\mathrm{O}$
$\mathrm{H}$
$\mathrm{O}$
$\mathrm{H}$
$\mathrm{O}$
$\mathrm{P}$
$\mathrm{P}$
$\mathrm{O}$
$\mathrm{H}$
$\mathrm{O}$
$\mathrm{H}$
$\mathrm{O}$
$\mathrm{O}$
$\mathrm{O}$
$\mathrm{H}$
$\mathrm{O}$
$\mathrm{H}$
$\mathrm{A}$

$\mathrm{Au}$

$$
\begin{array}{r}
0.03944300 \\
-0.91414000 \\
1.13618000 \\
-1.33933200 \\
0.38175400 \\
-1.80575100 \\
-1.31139000 \\
2.42674800 \\
3.13610900 \\
5.01118500 \\
3.11827400 \\
7.47999700 \\
6.96043100 \\
7.92718300 \\
-2.15589800 \\
-2.99691900 \\
-3.17463300 \\
8.21648600 \\
-4.21950500 \\
8.12025500 \\
8.90787600 \\
7.20123300 \\
6.29764200 \\
9.59209900 \\
-4.25980000 \\
-5.56281300 \\
-6.07723600 \\
-3.78422200 \\
-3.90842600 \\
-7.45011100
\end{array}
$$

$-0.38357500$

1.83119600

2.29389800

$-0.15787400$

$-1.24558300$

0.95746300

2. 67709300

$-2.02392300$

$-2.68154900$

$-2.09061000$

1.35447800

$-0.57518900$

3.91173100

3.89465600

$-1.05529900$

$-0.62761600$

1.17389300

$-1.77210800$

1.54743200

$-1.32678000$

$-0.85540300$

$-2.97187800$

$-2.65818400$

$-2.01009400$

0.53143000

1.79144300

0.97400500

3.02437800

3. 21431500

$-0.95157800$
0.71156700

$-0.68700400$

$-1.10204000$

0.65243100

1.26744500

$-0.06754600$

$-1.23794000$

0.86685600

1.02122100

0.78911800

$-0.20906400$

0.64721100

$-0.90162900$

$-0.84336800$

1.26759500

1.55059900

$-0.27804100$

$-0.20023500$

0.89656400

$-1.74642500$

$-2.06136700$

$-0.09473100$

0.27752200

0.30592400

1.99431900

0.08381400

$-0.12002700$

1. 32465700

2.26851900

$-0.55059900$

\section{Conformer 1(B)}

$\begin{array}{lrrr}\mathrm{C} & 3.19376300 & -1.84719300 & -1.89541700 \\ \mathrm{C} & 2.75880500 & -0.54228400 & -1.78159700 \\ \mathrm{C} & 1.43269000 & -0.23134300 & -1.36655800 \\ \mathrm{C} & 0.58158800 & -1.33570300 & -1.11737200 \\ \mathrm{C} & 1.00285200 & -2.66088700 & -1.22080400 \\ \mathrm{C} & 2.31678600 & -2.90918000 & -1.59727400 \\ \mathrm{H} & 4.21471700 & -2.03321200 & -2.21289700 \\ \mathrm{C} & 0.88359300 & 1.09380600 & -1.18123800 \\ \mathrm{H} & 0.32095700 & -3.47421600 & -1.00555500 \\ \mathrm{C} & -1.29507200 & 0.06625100 & -0.62065600 \\ \mathrm{C} & -0.52377100 & 1.18124200 & -0.83095600 \\ \mathrm{C} & -2.71039200 & -0.01934400 & -0.24264400 \\ & & & \end{array}$

Osonga et al

Page S15 
0.01256000

1. 12884500

$-0.13953200$

$-1.37426000$

0.37308600

$-2.18292900$

$-0.06181300$

1. 04585800

0.21748700

2. 11438700

$-0.33196600$

$-0.22588300$

0.48262600

$-2.33385400$

0.59277600

2. 43858700

$-0.69605700$

3. 01979500

$-0.85546000$

2.19652800

$-1.28630200$

$-1.16390500$

$-0.75923900$

0.46101100

$-2.17220200$

$-4.20583200$

$-1.68271000$

$-4.26561200$

$-1.90010100$

2. 20734000

0.32686400

2. 07892300

$-0.38779200$

1. 21688100

$-0.03125600$

1. 53885100

0.37410200

2.61630600

1. 38098700

$-0.50504300$

0.16437000

$-1.14903200$

$-0.80334100$

$-0.17542100$

2. 53545600

0.15212000

0.97324900

$-1.17657800$

0.13877200

$-2.16016400$

$-0.02592300$

$-0.93068800$

$-0.33427100$

1.14068500

1.80181000

$-1.00716500$

$-1.84300900$

2.01727300

$-1.05674000$

2. 31684400

$-2.71555500$

\section{Conformer 1(C)}

$\begin{array}{lrrr}\mathrm{C} & -2.69795500 & -0.99603500 & 1.16340500 \\ \mathrm{C} & -2.11141000 & 0.23791400 & 0.97980800 \\ \mathrm{C} & -0.73475900 & 0.36706900 & 0.63998800 \\ \mathrm{C} & 0.00602500 & -0.83853200 & 0.56688300 \\ \mathrm{C} & -0.57007900 & -2.09732700 & 0.73554900 \\ \mathrm{C} & -1.93191900 & -2.16939200 & 1.00755900 \\ \mathrm{H} & -3.75459300 & -1.04778000 & 1.40914300 \\ \mathrm{C} & -0.03854400 & 1.60123000 & 0.35733300 \\ \mathrm{H} & 0.02895400 & -2.99493800 & 0.64337100 \\ \mathrm{C} & 2.05760600 & 0.31298300 & 0.11612500\end{array}$

Osonga et al

Page S16 
C
C
C
C
C
H
C
H
C
H
O
H
O
$\mathrm{O}$
$\mathrm{O}$
$\mathrm{O}$
$\mathrm{H}$
$\mathrm{O}$
$\mathrm{H}$
$\mathrm{O}$
$\mathrm{P}$
$\mathrm{P}$
$\mathrm{O}$
$\mathrm{H}$
$\mathrm{O}$
$\mathrm{O}$
$\mathrm{O}$
$\mathrm{O}$
$\mathrm{H}$
$\mathrm{O}$
$\mathrm{H}$
Au
$\mathrm{H}$

$$
\begin{array}{r}
1.39443500 \\
3.48219000 \\
3.93793100 \\
4.41190500 \\
5.28365200 \\
3.24418600 \\
5.77185000 \\
4.10662100 \\
6.19076600 \\
5.64973500 \\
2.01337500 \\
1.29603200 \\
-0.54173600 \\
1.33914100 \\
-2.86942100 \\
-2.48439000 \\
-3.44822200 \\
6.59019000 \\
7.50756900 \\
7.50840500 \\
-3.84816100 \\
8.79646700 \\
-4.98317300 \\
-4.69589900 \\
-4.40518000 \\
-2.96088100 \\
8.96771200 \\
9.97053500 \\
10.31235400 \\
8.66015600 \\
8.98522300 \\
-5.85595100 \\
-1.98442900
\end{array}
$$

1.51436600

0.05134700

$-1.28109800$

1.09971100

$-1.53599300$

$-2.10692900$

0.85173500

2.13310700

$-0.48536800$

$-2.55153900$

2. 69668200

3. 36406400

2. 75860600

$-0.83327400$

1.36299600

$-3.39897200$

$-3.33212300$

1. 92431600

1. 71387100

$-0.81695600$

2.07702900

$-0.52885400$

2. 74321600

3. 55822100

1. 12337800

3. 28035300

0.89945700

$-1.17224600$

$-0.57388900$

$-1.56882800$

$-1.20736600$

$-0.95063200$

3.13713700
0.13747500

$-0.11895700$

$-0.22663100$

$-0.22840300$

$-0.45646400$

$-0.14079000$

$-0.45136900$

$-0.14517100$

$-0.57667600$

$-0.56470700$

$-0.08448900$

$-0.06219900$

0.26931000

0.31489500

1.22433500

1.13875200

1.20626500

$-0.57712700$

$-0.29776000$

$-0.93781100$

0.10985300

$-0.02110700$

1.02366600

1.46099800

$-0.88506200$

$-0.40417200$

0.37164200

$-0.89623800$

$-1.57718500$

1.18936600

2.02743500

$-0.51872900$

$-0.16131300$
Conformer 1(D)

$\mathrm{C}$
$\mathrm{C}$
$\mathrm{C}$
$\mathrm{C}$
$\mathrm{C}$
$\mathrm{C}$
$\mathrm{H}$
$\mathrm{C}$
$\mathrm{H}$

$\mathrm{C}$

C

C

C

C

C

$\mathrm{H}$

C

$\mathrm{H}$

C
5.22772000

4.70276400

3.30133800

2.48274700

2.99463000

4.37256200

6.30201800

2.65064600

2.33366400

0.46453600
2. 25672100

1. 24704100

0.99241500

1.85765100

2.87875200

3.06306700

2. 41062100

$-0.05819100$

3.50937200

0.79844600
$-0.55078400$

0.23162700

0.27160700

$-0.49585600$

$-1.29399900$

$-1.32716800$

$-0.55441300$

1.02293900

$-1.87565200$

0.23681200

Osonga et al

Page S17 
C
C
C
C
C
H
C
H
C
H
O
H
O
O
$\mathrm{O}$
$\mathrm{O}$
H
O
H
O
P
P
$\mathrm{O}$
$\mathrm{O}$
$\mathrm{O}$
$\mathrm{O}$
$\mathrm{O}$
$\mathrm{H}$
$\mathrm{O}$
A
$\mathrm{H}$
$\mathrm{H}$
$\mathrm{H}$

C

C

C

0

H

H

$P$

P

(

O

$\mathrm{H}$

$\mathrm{Au}$

$\mathrm{H}$

$\mathrm{H}$

$\mathrm{H}$

\section{Conformer 1 (E)}

$$
\begin{array}{r}
1.19516900 \\
-0.98952200 \\
-1.55023500 \\
-1.86053500 \\
-2.93150400 \\
-0.90710600 \\
-3.24631300 \\
-1.45899100 \\
-3.78653900 \\
-3.36994700 \\
0.62822300 \\
1.38962500 \\
3.19626800 \\
1.12970900 \\
5.55216600 \\
4.84866600 \\
5.81531700 \\
-4.12193300 \\
-3.63568600 \\
-5.14839400 \\
6.35986100 \\
-6.20047800 \\
6.48102700 \\
5.60358600 \\
7.76983300 \\
-6.84135000 \\
-7.40783100 \\
-7.91887000 \\
-5.60837200 \\
-1.69617600 \\
4.65474300 \\
-6.13563200 \\
7.72042900
\end{array}
$$

$-0.08179600$

0.88883500

1. 88927100

$-0.00575400$

2. 01126200

2.57984200

0.13396100

$-0.73776000$

1.14640800

2.78485300

$-1.05945400$

$-1.54726700$

$-0.96710300$

1.73889500

0.54579900

4.05540100

4.07335200

$-0.69298600$

$-1.38704200$

1.25968100

$-0.82448200$

1.64311400

$-0.94358300$

$-1.93677500$

$-0.67338300$

0.21775100

2. 31479800

1. 68875700

2.47825900

$-2.37338700$

$-1.65242500$

$-0.41492000$

$-0.89625400$
0.99087100

0.08410500

$-0.73147800$

0.75327700

$-0.85198000$

$-1.26075900$

0.63101400

1. 44306900

$-0.17317900$

$-1.47318500$

1.73652300

2.11922700

1.70631400

$-0.49923800$

1. 04880600

$-2.11984400$

$-2.10139800$

1.29191500

1.75812500

$-0.36326400$

0.57911900

0.84639200

$-0.88624800$

1. 42698000

1.33364500

1.23878800

0.04004100

$-0.49408800$

1.90620100

$-1.01195400$

1.58921000

1. 46500600

2. 27481000
C

C

C

C

C

C

$\mathrm{H}$

C

$\mathrm{H}$

C

C

C

C
5.78000600

5.71298500

4.49108700

3.35236000

3.40021900

4.62244400

6.72414800

4.30077000

2.50100700

1.88756700

2.94690200

0.47680900

$-0.40275900$
2. 38628200

1.01100600

0.30689000

1.10689900

2.49496900

3.13030200

2.88508700

$-1.12252800$

3.05893600

$-0.78388000$

$-1.62006400$

$-1.12672600$

$-0.13607900$
0.38399900

0.47683300

0.26701200

$-0.00343000$

$-0.11557400$

0.07010700

0.57501700

0.32422600

$-0.33177700$

$-0.05749500$

0.19472300

$-0.26875800$

$-0.74787500$

Osonga et al

Page S18 
$-0.02192500$

$-1.74725000$

$-0.05141300$

$-1.36883000$

0.63230900

$-2.22856400$

2. 80958800

3.71867200

5.20354200

2.12500700

6.84717100

4.64829700

5.54007400

$-3.56880200$

8.06026500

$-4.73724500$

8.11283400

8.75849000

7.48471300

9.31787400

$-4.69987400$

$-4.19969500$

$-3.92554500$

$-5.99870100$

$-5.84091900$

6.56612600

$-1.75264600$

$-2.55640000$

$-3.44963800$

$-5.08961900$
$-2.42014600$

$-0.42508800$

0.86437500

$-2.70781400$

$-3.19911700$

$-1.71480900$

$-2.96518700$

$-3.30902700$

$-2.00417000$

0.55403300

0.34704300

4.47935900

4.82630400

$-1.95377600$

$-0.27430400$

$-2.38266500$

0.61751100

1. 34033300

$-1.64573100$

$-0.29944300$

$-1.31509200$

$-3.69853900$

$-3.57355200$

$-2.55904700$

1.66543200

$-1.84917900$

$-3.70400500$

0.55586700

0.20397100

$-0.43661700$
$-0.00827200$

$-0.96380000$

$-0.96719300$

$-0.22382800$

0.35472000

$-0.68412300$

0.28422600

0.40742100

0.46850700

$-0.16435100$

0.90364200

$-0.04115400$

0.11000300

$-0.99097700$

$-0.01048500$

0.06622200

$-1.35079900$

$-1.30105800$

$-0.52999700$

0.77967700

1.26062300

0.80470200

1.72749000

$-0.68942400$

0.16656500

$-0.11512100$

$-0.03795900$

$-1.44988100$

$-1.59204900$

1.03502000

\section{Conformer 1(F)}

\begin{tabular}{lrrr} 
C & 6.93178500 & 1.48436400 & -0.49796600 \\
$\mathrm{C}$ & 6.29928200 & 0.25883000 & -0.44582800 \\
$\mathrm{C}$ & 4.87982600 & 0.15385200 & -0.36191000 \\
$\mathrm{C}$ & 4.16458700 & 1.37774200 & -0.37964300 \\
$\mathrm{C}$ & 4.78324600 & 2.62577900 & -0.41807100 \\
$\mathrm{C}$ & 6.17159800 & 2.67318400 & -0.46609700 \\
$\mathrm{H}$ & 8.01253900 & 1.51944600 & -0.58474700 \\
$\mathrm{C}$ & 4.10996200 & -1.06429000 & -0.28296600 \\
$\mathrm{H}$ & 4.18980500 & 3.53196700 & -0.42092200 \\
$\mathrm{C}$ & 2.04147100 & 0.27618300 & -0.36344000 \\
$\mathrm{C}$ & 2.66826700 & -0.94501200 & -0.33220900 \\
$\mathrm{C}$ & 0.60467700 & 0.57242100 & -0.36478200 \\
$\mathrm{C}$ & -0.35252300 & -0.44112900 & -0.56738100 \\
$\mathrm{C}$ & 0.16959100 & 1.90058600 & -0.16938800 \\
$\mathrm{C}$ & -1.71190000 & -0.13530500 & -0.56649500 \\
$\mathrm{H}$ & -0.05821000 & -1.46870000 & -0.72802800 \\
$\mathrm{C}$ & -1.18892800 & 2.20609700 & -0.17039500 \\
$\mathrm{H}$ & 0.88918300 & 2.69444800 & -0.01743700 \\
\hline
\end{tabular}

Osonga et al

Page S19 


$$
\begin{array}{r}
-2.12148500 \\
1.98438400 \\
2.66915900 \\
4.56567900 \\
2.80925500 \\
7.08298900 \\
6.75361200 \\
7.71892100 \\
-3.49756000 \\
7.82149800 \\
-4.33817900 \\
6.65642300 \\
5.78998900 \\
8.18322300 \\
8.98159700 \\
-4.06247700 \\
-5.82953500 \\
-6.06808400 \\
-4.04018600 \\
-6.52978900 \\
9.08693600 \\
-1.52719200 \\
-2.61155700 \\
-3.51402900 \\
-4.08895600
\end{array}
$$

\section{Conformer $1(\mathrm{G})$}

\begin{tabular}{lr}
$\mathrm{C}$ & -5.88355000 \\
$\mathrm{C}$ & -5.76676900 \\
$\mathrm{C}$ & -4.52199500 \\
$\mathrm{C}$ & -3.41454400 \\
$\mathrm{C}$ & -3.51288900 \\
$\mathrm{C}$ & -4.75565900 \\
$\mathrm{H}$ & -6.84330900 \\
$\mathrm{C}$ & -4.28020200 \\
$\mathrm{H}$ & -2.63596200 \\
$\mathrm{C}$ & -1.88297100 \\
$\mathrm{C}$ & -2.90975100 \\
$\mathrm{C}$ & -0.46066600 \\
$\mathrm{C}$ & 0.38024300 \\
$\mathrm{C}$ & 0.08726100 \\
$\mathrm{C}$ & 1.73672100 \\
$\mathrm{H}$ & -0.00833400 \\
$\mathrm{C}$ & 1.44393900 \\
$\mathrm{H}$ & -0.53568000 \\
$\mathrm{C}$ & 2.26109800 \\
$\mathrm{O}$ & -2.72300200 \\
$\mathrm{H}$ & -3.61795600 \\
$\mathrm{O}$ & -5.14995700 \\
$\mathrm{O}$ & -2.16896800 \\
\hline
\end{tabular}

$$
\begin{array}{rr}
2.33139600 & -0.34074500 \\
0.96171500 & -0.46283200 \\
0.29693900 & -0.25844500 \\
1.13111300 & 0.03781700 \\
2.51361600 & 0.18054600 \\
3.10900900 & -0.00094700 \\
2.80050200 & -0.52938500 \\
-1.12329000 & -0.34434200 \\
3.10435100 & 0.41601900 \\
-0.70623100 & 0.05855100 \\
-1.57460200 & -0.21752600 \\
-1.00192200 & 0.26632700 \\
0.00949200 & 0.76932300 \\
-2.26717100 & -0.03151400 \\
-0.23009000 & 0.97631200 \\
0.99144800 & 1.00851300 \\
-2.50804700 & 0.17759900 \\
-3.05884100 & -0.42111800 \\
-1.49606600 & 0.67116100 \\
-2.91136800 & -0.33620000 \\
-3.28536300 & -0.47398800 \\
-2.03324500 & -0.51276600 \\
0.61930300 & 0.19396000
\end{array}
$$$$
-0.35965800
$$$$
-0.28834700
$$$$
-0.21022000
$$$$
-0.16280000
$$$$
-0.36818800
$$$$
-0.60693400
$$$$
-0.50310200
$$$$
-0.53950400
$$$$
-0.42364600
$$$$
0.55576500
$$$$
0.64917800
$$$$
1.18460100
$$$$
0.63733400
$$$$
1.74659000
$$$$
-0.03441300
$$$$
2.00596000
$$$$
0.17849700
$$$$
0.12602600
$$$$
0.70166300
$$$$
-0.08676300
$$$$
1.68546700
$$$$
-0.02513400
$$$$
-0.77059700
$$$$
-0.74231200
$$$$
2.80268600
$$

\section{Osonga et al}

Page S20 
$-0.91414800$

0.13974800

$-0.01174900$

0.98033800

$-0.02438000$

$-0.10551200$

1.32548100

1.27357900

0.48935600

$-0.83374000$

$-1.16740300$

$-0.61595200$

$-1.47834500$

0.84429900

$-0.23256500$

0.07208800

$-0.04179000$

1.47964100

1.52315100

1.51541100

\section{Conformer $1(\mathrm{H})$}

C
C
C
C
C
C
H
C
H
C
C
C
C
C
C
H
C
H
C
O
H
O
O
O
O
H

C

$\mathrm{H}$

$\mathrm{H}$
3.59732600

3.37504200

2. 06532300

1.01207300

1.21503600

2. 51409000

4.60692200

1. 71080300

0.37465000

$-0.66815600$

0.30017600

$-2.12454400$

$-2.73416300$

$-2.94095000$

$-4.12188100$

$-2.14671900$

$-4.32022800$

$-2.50176500$

$-4.90862400$

0.00443900

0.86321300

2.50879100

$-0.28008000$

4.45500200

2.68877100

3.62361200
3.89371200

2.56396700

2. 06449900

3.00849700

4.35816300

4.79775900

4.22698000

0.70098300

5.03971400

1. 36004000

0.39177300

1.23127500

$-0.00154000$

2.36992800

$-0.11588000$

$-0.89816600$

2. 26330100

3. 33081100

1.03808600

$-0.90445600$

$-1.37727900$

$-0.26862600$

2.64300600

1.70698800

6.11169500

6.31728800
$-0.77833800$

$-0.48268200$

$-0.22181400$

$-0.31431300$

$-0.59567300$

$-0.82076000$

$-0.99575400$

0.09494500

$-0.64667800$

0.11521300

0.22002400

0.24623600

0.54151600

0.06946500

0.67381000

0.67796600

0.20014000

$-0.16363000$

0.50736400

0.47774500

0.50950800

0.27586500

$-0.13344300$

$-0.56509700$

$-1.09706700$

$-1.24517500$

\section{Osonga et al}

Page S21 
0.75416100

1. 26286900

0.62574800

0.21054100

$-0.11393700$

0.00821500

1.27767300

$-0.15285100$

0.87797400

2.39418200

1.76564200

1.07688600

0.07694800

1.00004300

$-1.49957400$

0.63558800

0.58244700

0.15521900

1. 40206300

$-1.25355800$

$-0.19779600$

$-4.13018500$

$-0.33847300$

3.10117400

1. 68432400

3.13284700

0.08335200

$-1.33671800$

1.00099400

$-1.43960500$

0.66864200

0.43663700

$-2.26916700$

Figure S2: Computed optimized QDP - gold structures and energies for conformersA to G.
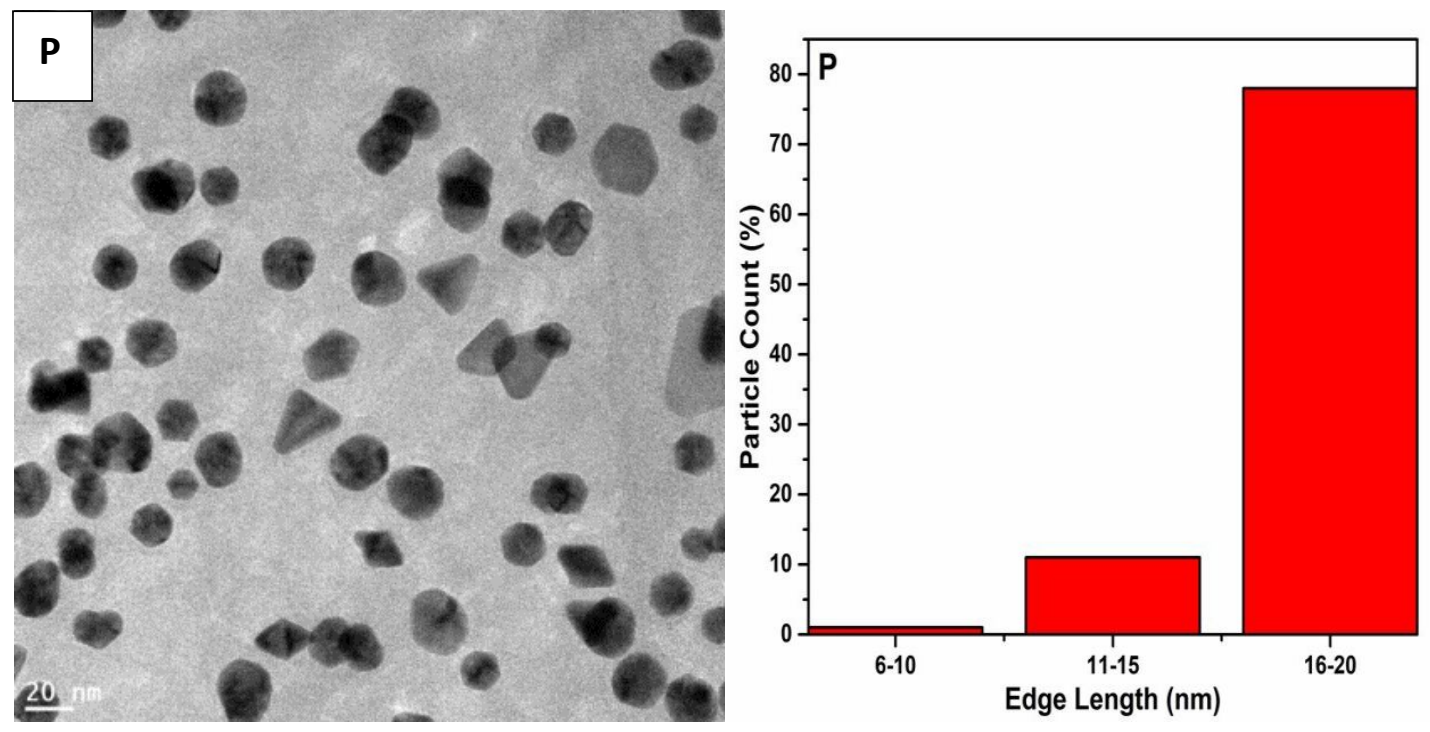

Osonga et al

Page S22 

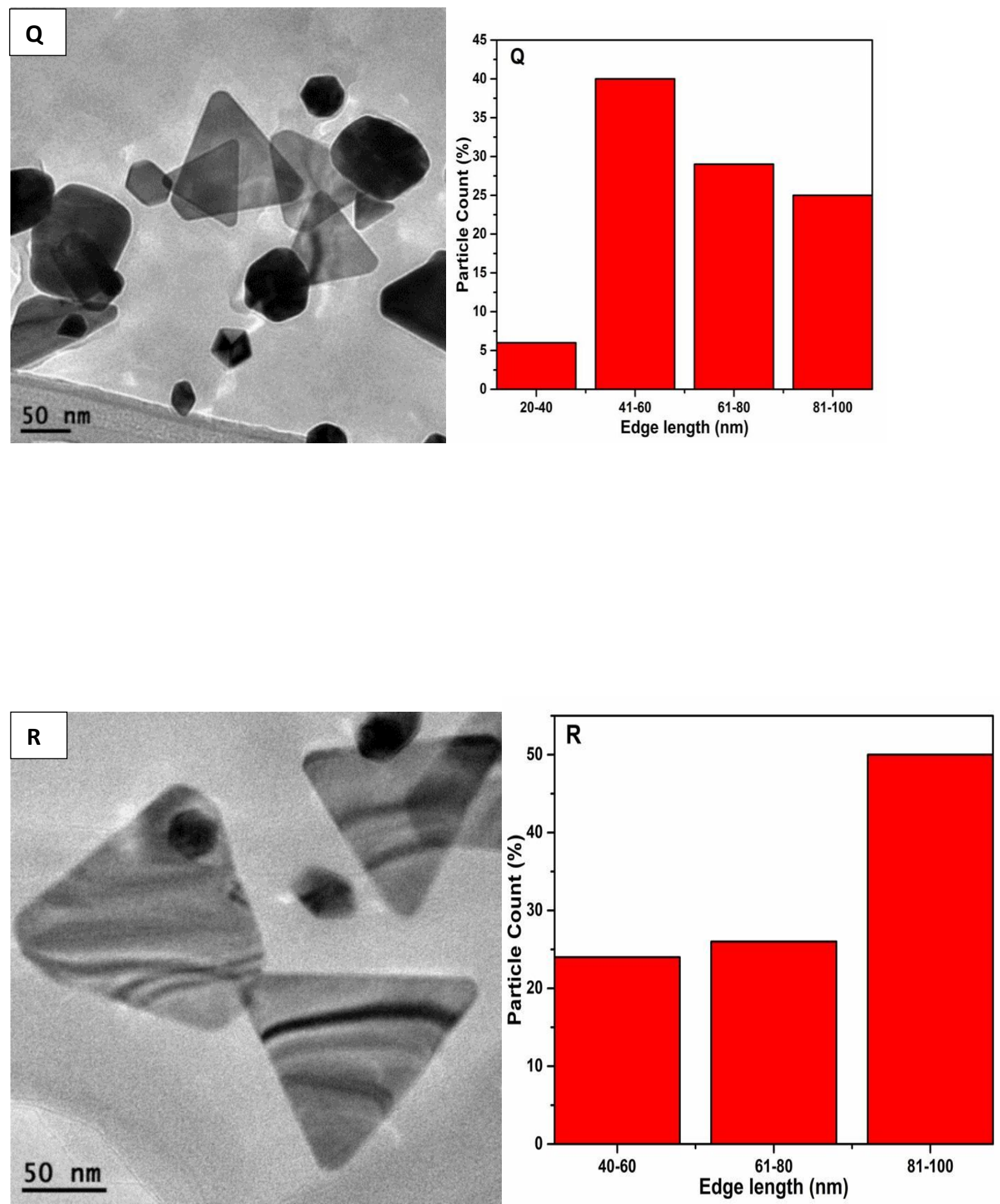

Osonga et al Page S23 

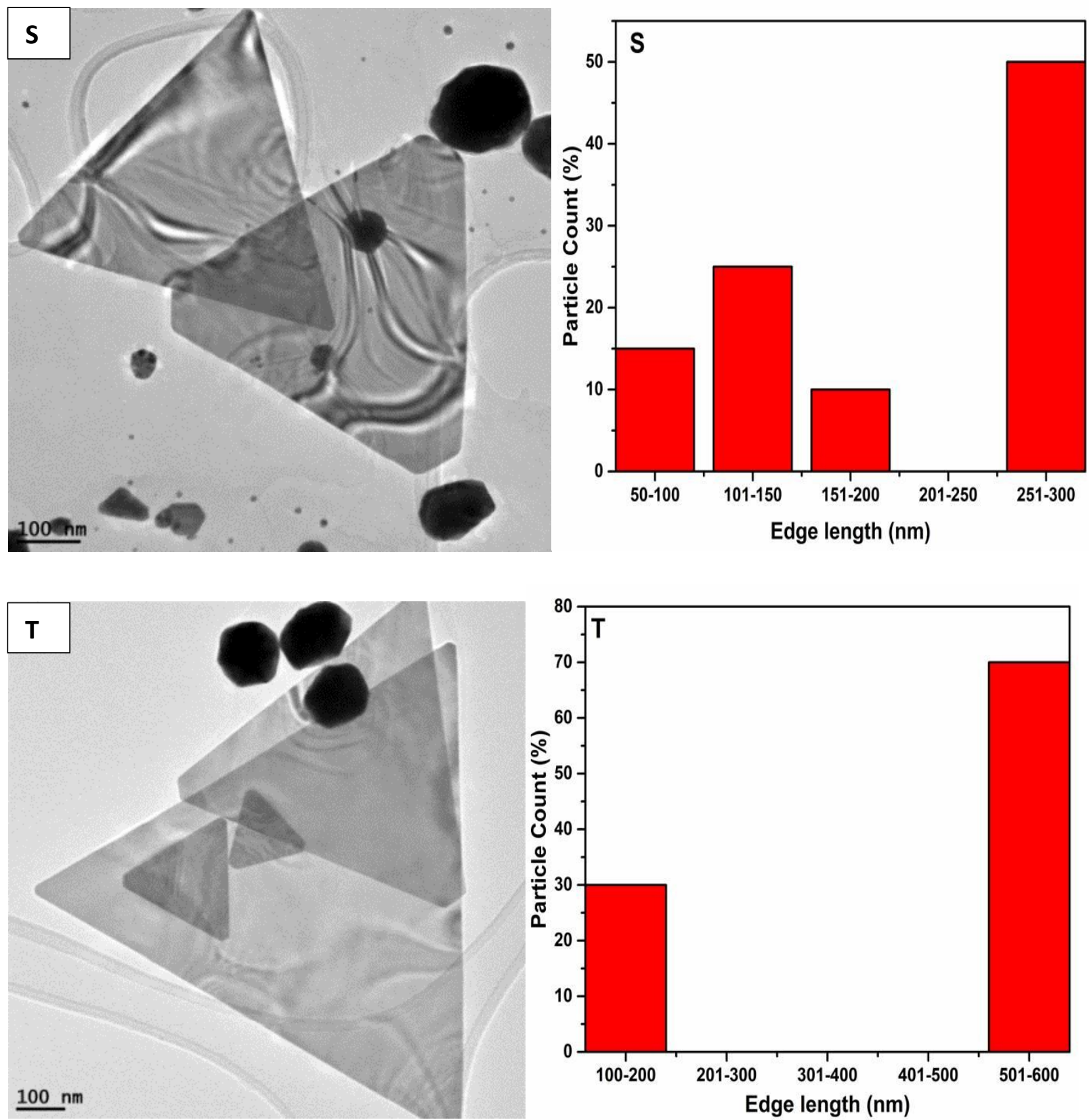

Osonga et al Page S24 

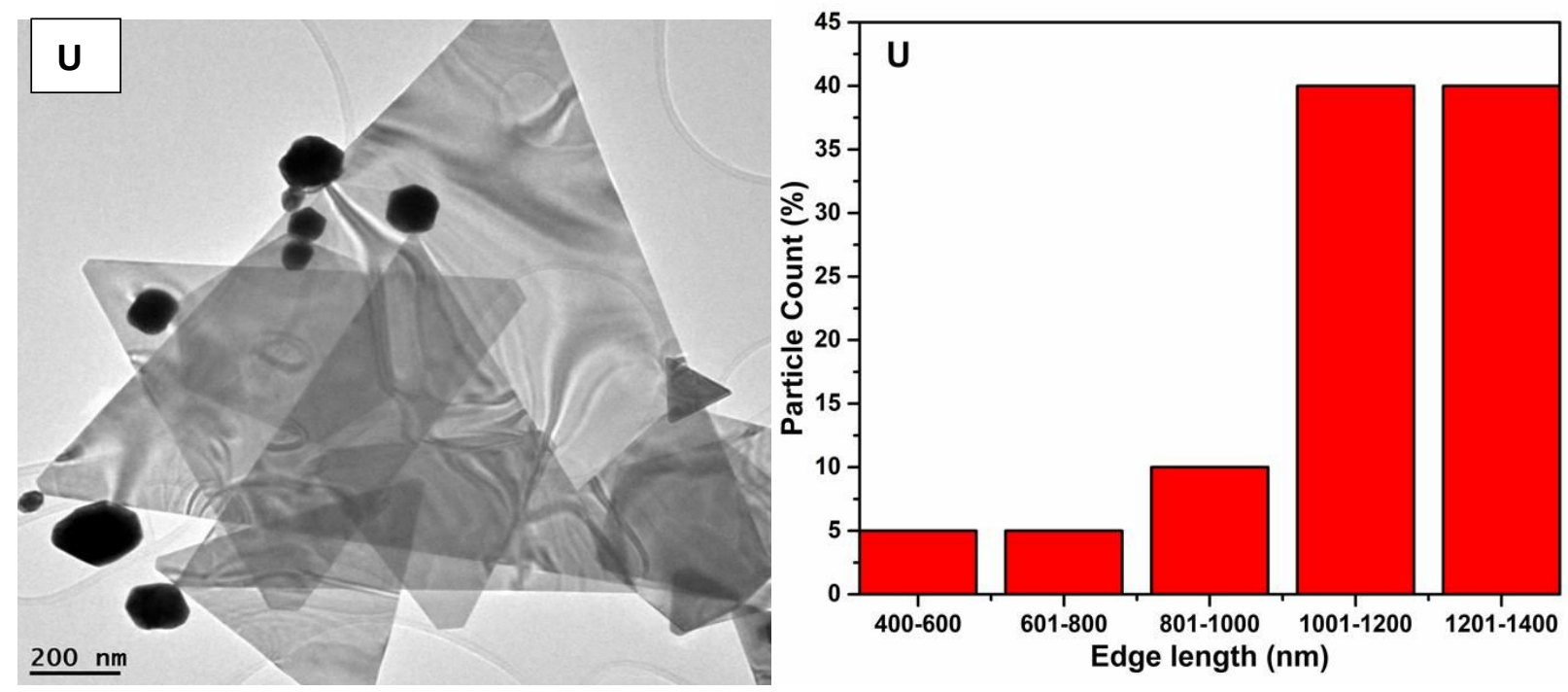

Figure S3:TEM images for the samples $\mathrm{P}, \mathrm{Q}, \mathrm{R}, \mathrm{S}, \mathrm{T}$ and $\mathrm{U}$ and the corresponding histogram showing edge length distribution of gold nanoplatesformed after 55 minutes. 